\title{
Low expression of miR-199 in hepatocellular carcinoma contributes to tumor cell hyper-proliferation by negatively suppressing XBP1
}

\author{
ZHENG LOU ${ }^{1 *}$, YONG-QIANG GONG ${ }^{2 *}, \mathrm{XING}^{\mathrm{ZHOU}}{ }^{2}$ and GUO-HUANG HU ${ }^{2}$ \\ ${ }^{1}$ Key Laboratory Breeding Base of Hunan Oriented Fundamental and Applied Research of Innovative Pharmaceutics, \\ Changsha Medical University, Changsha, Hunan 410219; ${ }^{2}$ Department of General Surgery, Institute of Digestive Surgery of \\ Changsha, Affiliated Changsha Hospital of Hunan Normal University, Changsha, Hunan 410006, P.R. China
}

Received November 17, 2017; Accepted August 16, 2018

DOI: $10.3892 / \mathrm{ol} .2018 .9476$

\begin{abstract}
Hepatocellular carcinoma (HCC) is the third leading cause of cancer-related mortality worldwide, and microRNAs (miRs) are considered to serve important functions in the pathogenesis of HCC by regulating the expression of specific target genes. The present study was conducted to investigate the role of miR-199 and its putative target X-box binding protein 1 (XBP1) in HCC, as well as of the downstream gene cyclin D. The expression levels of miR-199, XBP1 and cyclin D were detected in clinical HCC specimens. The effect of miR-199 on the regulation of HCC cell proliferation and its underlying mechanism were examined in Hep3B2.1-7 cells, through expression assays and measurement of cell proliferation (via Cell Counting Kit-8, and 5-ethynyl-2'-deoxyuridine and DAPI double-staining assays) coupled with gain- and lose- of function experiments. The expression of XBP1 and cyclin D was significantly increased in HCC tissues when compared with adjacent non-HCC tissues, while the expression of miR-199 was decreased. Exogenous miR-199 significantly suppressed the expression of XBP1 and cyclin D in Hep3B2.1-7 cells. However, the expression of XBP1 and cyclin D significantly increased on treatment with miR-199 inhibitor. Consistently, Hep3B2.1-7 cells co-transfected with a wild type reporter plasmid [XBP1-3'untranslated region (UTR)-WT] and exogenous miR-199 exhibited lower relative luciferase enzyme activity than cells co-transfected with negative control
\end{abstract}

Correspondence to: Dr Guo-Huang Hu, Department of General Surgery, Institute of Digestive Surgery of Changsha, Affiliated Changsha Hospital of Hunan Normal University, 70 Lu-Shan Road, Changsha, Hunan 410006, P.R. China

E-mail: hgh123416@163.com

${ }^{*}$ Contributed equally

Key words: liver cancer, hepatocellular carcinoma, cell proliferation, microRNAs, microRNA-199, X-box binding protein 1, cyclin D
miRNA and XBP1-3'UTR-WT, while cells co-transfected with mutated plasmid (XBP1-3'UTR-MU) and miR-199 exhibited no change. It was further observed that knockdown of XBP1 by small interfering RNA significantly decreased the expression of cyclin D in Hep3B2.1-7 cells. Additionally, exogenous miR-199 decreased the proliferation of Hep3B2.1-7 cells, which was contrary to the effect of miR-199 inhibitor. In conclusion, it was demonstrated that miR-199 negatively regulated the expression of XBP1 by directly binding to its 3'UTR and that XBP1 impacted cyclin D expression, which was associated with the cell cycle regulation in Hep3B2.1-7 cells. These findings suggested that a miR-199/XBP1/cyclin D axis may serve an important role in the pathogenesis of HCC.

\section{Introduction}

Hepatocellular carcinoma (HCC) is the dominant form of primary liver cancer, accounting for more than $80 \%$ of all liver cancer cases, and is the third leading cause of cancer-related mortality worldwide $(1,2)$. Despite advances in prevention techniques, screening and novel technologies used in the diagnosis and treatment of HCC, incidence and mortality rates continue to rise $(1,2)$. Previous reports describe HCC as a type of heterogeneous disease, and surgical resection and chemotherapy are the common treatment methods; however, postoperative recurrence and drug resistance pose challenges for the treatment of HCC (1-3). To date, sorafenib is the only drug approved by the US Food and Drug Administration as a molecular targeting therapy for HCC, but only extends the survival time of patients by $2-3$ months $(2,3)$. Research has demonstrated that hyper-proliferation of tumor cells is the main biological basis of tumor migration, diffusion, invasion, postoperative recurrence and drug resistance (4-6). Although progress has been made in research on the regulation underlying tumor cell proliferation regulation, the mechanisms are still largely unknown and need to be elucidated.

$\mathrm{X}$-box binding protein 1 (XBP1), a member of the basic leucine zipper family of transcription factors CREB/ATF (cyclic adenosine monophosphate response element binding protein/activation of transcription factors), is encoded by the XBP1 gene and widely expressed in various tissues in adult 
mammals, with particularly high expression in the exocrine glands and liver, and is a necessary factor for liver cell differentiation and liver formation (7). Dysregulation of XBP1 has been associated with tumor pathogenesis. In particular, researches have reported that the inositol-requiring enzyme $1 \alpha$ $(\mathrm{IRE} 1 \alpha) / \mathrm{XBP} 1$ pathway is involved in all processes associated with the development and progression of tumors, including tumor cell survival and proliferation, epithelial-mesenchymal transition, transcription factor regulation, drug resistance and glycolysis (8-13). In addition, several studies have reported cyclin D to be a downstream effector of XBP1 (13-15), which in turn is established as an important regulator of the cell cycle; previous study demonstrated that inhibition of cyclin D induced cell cycle arrest at G1 phase, suggesting that cyclin D is involved in regulation of cell proliferation (13). However, the detailed mechanism for the upstream regulation of cyclin D is yet to be fully elucidated.

MicroRNAs (miRNA/miRs) are a class of endogenous non-coding small RNAs (20-24 nucleotides in length) that negatively regulate protein expression via mRNA degradation or translation inhibition, and are widely involved in cell proliferation, differentiation and apoptosis, which can have important impacts in the pathogenesis of tumors (16). Previous studies demonstrated that the expression levels of several miRNAs were significantly altered in HCC cancer cells, and indicated involvement of the miRNAs in the pathophysiological processes of HCC development; for instance miR-503a, -138, -199 and -214 were associated with cell cycle regulation (16-19). Preliminary experiments by our group found that miR-199 expression was significantly decreased in HCC tumor cells, and implicated XBP1 as a putative target of miR-199 (unpublished data). Considering XBP1 serves an important role in cell cycle regulation, we speculate that miR-199 may participate in the process of HCC cell proliferation by regulating the expression of XBP1, and in turn cyclin D. However, there is a lack of research on the role of miR-199/XBP1 in HCC at present.

The present study aimed to investigate whether miR-199 is involved in the regulation of HCC tumor cell proliferation and its potential underlying molecular mechanism. To our knowledge, the present study is the first to investigate the role of an miR-199/XBP1/cyclin D pathway in cell cycle regulation, and may a provide novel theoretical basis for HCC treatment and drug development.

\section{Materials and methods}

Clinical samples. Liver cancer and normal para-carcinoma tissue samples from patients (including 6 females and 9 males enrolled between September 2014 and November 2017; age range, 46-78 years) diagnosed with $\mathrm{HCC}$ were provided by the Department of General Surgery of Affiliated Changsha Hospital of Hunan Normal University, Changsha, China. Prior to experiments, the tumor tissue and its peripheral normal tissue were frozen in liquid nitrogen. The study was performed following the guidelines of the Declaration of Helsinki and the experiments were approved by the Hunan Normal University Medical Ethics Committee, and all patients had signed written informed consent.

The tissues were allocated to two groups ( $n=15$ per group): A control group (normal para-carcinoma tissues) and a HCC group (cancer tissues). The frozen liver tissues were used evaluate the expression of mRNA and protein.

Cell culture. The HCC cell line Hep3B2.1-7 was purchased from the Cell Bank of Type Culture Collection of Chinese Academy of Sciences (Shanghai, China). Cells were cultured with Dulbecco's modified Eagle's medium (DMEM) supplemented with $10 \%$ fetal bovine serum in a carbon dioxide incubator at $37^{\circ} \mathrm{C}$ in $5 \% \mathrm{CO}_{2}$. A total of $1 \times 10^{4}$ cells were subcultured and seeded into 6 or 24 -well plates for mimic and small interfering (si)RNA transfection experiments. Following these, cells were digested with $0.2 \%$ trypsinogen and the lysates collected for mRNA and protein analyses.

Bioinformatics analysis. The target genes of miRNA and the cognate miRNAs of a gene were predicted using the online systems TargetScan (http://www.targetscan.org/vert_72/) and miRBase (http://www.mirbase.org/). Focus was on the upregulated XBP1 gene and the downregulated miR-199 in HCC tissue, due to them being potential targets of each other, according to the bioinformatics analysis.

miR-199 mimic/inhibitor transfection. miR-199 mimic and inhibitor transfection experiments were conducted with Lipofectamine 2000 according to the manufacturer's instructions. Prior to transfection, a mix including Lipofectamine 2000 and miR-199 mimic (5'-CCCAGUGUUUAGACUAUC UGUUC-3'; $50 \mu \mathrm{M}$ ) and inhibitor (5'-GGGUCACAAAUC UGAUAGACAAG-3'; $100 \mu \mathrm{M}$ ), or negative control (NC) miRNA (5'-UCACAACCUCCUAGAAAGAGUAGA-3'; $50 \mu \mathrm{M})$ were prepared. The mix was then added to the 6 or 24-well plates with cultured Hep3B2.1-7 cells to make a final concentration of $80 \mathrm{nmol} / 1$ for miR-199 mimics and $\mathrm{NC}$ miRNA, and $160 \mathrm{nmol} / 1$ for miR-199 inhibitors, and the cells were transfected at $37^{\circ} \mathrm{C}$ for $6 \mathrm{~h}$ prior to a $24 \mathrm{~h}$ normal culture at $37^{\circ} \mathrm{C}$. Subsequently, the cells were used for proliferation, mRNA and protein analyses.

Luciferase assay. The XBP1 sequence (Shanghai Genechem Co., Ltd., Shanghai, China) containing the seed sequence (5'-ACACUGGG-3') of the putative binding site for miR-199 or the identical sequence with a mutation of the miR-199 seed sequence (5'-ACACCGGG-3') was inserted between the restriction sites of XhoI and NotI of a pmiR-RB-Report ${ }^{\mathrm{TM}}$ luciferase vector (Promega Corporation, Madison, WI, USA) and validated by sequencing. Hep3B2.1-7 cells were transfected with the mix of Lipofectamine 2000 and the wild type or mutated reporter vectors and miR-199 mimic or NC (5'-UCACAACCUCCU AGAAAGAGUAGA-3') at $37^{\circ} \mathrm{C}$ for $6 \mathrm{~h}$. Lysates were harvested $24 \mathrm{~h}$ after transfection using a specific report gene cell lysis fluid (Beyotime Institute of Biotechnology, Shanghai, China). Renilla luciferase activities were measured according to the protocol of the Dual-Luciferase Assay System (Promega Corporation).

XBP1 siRNA transfection. An XBP1 siRNA transfection experiment was conducted according to the instructions of a XBP-1 siRNA(h) kit (sc-8015; Santa Cruz Biotechnology, Inc., Dallas, TX, USA). Prior to transfection, a mix including transfection agent Entranster ${ }^{\mathrm{TM}}-\mathrm{R} 4000$ and XBP1 siRNA (sense, 5'-TGCCAATGAACTCTTTCCC-3', and antisense, 5'-CCA 
Table I. Primers for real-time reverse transcription-quantitative polymerase chain reaction.

Primer, 5'-3'

\begin{tabular}{lllc}
\cline { 2 - 3 } Gene & \multicolumn{1}{c}{ Forward } & \multicolumn{1}{c}{ Reverse } & Product size, bp \\
\hline XBP1 & TCCTGTTGGGCATTCTGGAC & GGCTGGTAAGGAACTGGGTC & 122 \\
Cyclin D & TGGCAAGCGTGTCATTGTTG & GGGGCTCACAGTAAGACTGG & 188 \\
$\beta$-actin & CCGTTCCGAAAGTTGCCTTT & ATCATCCATGGTGAGCTGGC & 170 \\
miR-199 & CTGAGTCCCAGTGTTCAGACT & GTGCAGGGGTCCGAGGT & 24 \\
U6 & CTCGCTTCGGCAGCACA & AACGCTTCACGAATTTGCGT & 94 \\
\hline
\end{tabular}

XBP1, X binding protein 1; miR, microRNA.

CATATATACCAAGCCC-3') or NC siRNA (sense, 5'-UUC UCCGAACGUGUCACGUTT-3', and antisense, 5'-ACGUGA CACGUUCGGAGAATT-3') were prepared. The mix was then added to the 6 or 24-well plates with cultured Hep3B2.1-7 cells to make a final concentration of 2 pmol/1 XBP1 siRNA or NC siRNA, and the cells were transfected at $37^{\circ} \mathrm{C}$ for $48 \mathrm{~h}$ prior to a $24 \mathrm{~h}$ normal culture at $37^{\circ} \mathrm{C}$. Subsequently, the cells were collected for mRNA and protein analyses.

Measurement of cell proliferation. To observe the proliferation of Hep3B2.1-7 cells treated with miR-199 mimic or inhibitor, cell numbers were determined with a Cell Counting Kit-8 (CCK-8) at 12, 24 and $36 \mathrm{~h}$ after transfection, according to the manufacturer's instructions. Optical density (OD) values were measured at $490 \mathrm{~nm}$ with a microplate reader, with the OD values being proportional to the number of cells.

5-Ethynyl-2'-deoxyuridine (EdU) and DAPI double staining. EdU staining was conducted with a BeyoClick ${ }^{\mathrm{TM}}$ EdU Cell Proliferation kit with Alexa Fluor 555 (Beyotime Institute of Biotechnology). According to the manufacturer's instructions, Hep3B2.1-7 cells were firstly labeled with $50 \mu \mathrm{M}$ EdU solution and incubated for $2 \mathrm{~h}$, then fixed with $4 \%$ paraformaldehyde for $30 \mathrm{mins}$ and neutralized with $2 \mathrm{mg} / \mathrm{ml}$ glycine. Subsequently, $0.5 \%$ Triton X-100 was added to increase cell membrane permeability, and then the Edu staining reaction solution was added and incubated in the dark at room temperature for 30 mins. Finally, 1X DAPI (Beyotime Institute of Biotechnology) staining solution was added and incubated in the dark for another 30 mins, and the Hep3B2.1-7 cells were imaged with a confocal microscope.

Real-time reverse transcription-quantitative polymerase chain reaction ( $R T-q P C R)$. Total RNA was extracted from the liver tissues samples or Hep3B2.1-7 cells by using TRIzol reagent (Takara Biotechnology Co., Ltd., Dalian, China) and the concentration and purity of RNA were determined spectrophotometrically. A total $200 \mathrm{ng}$ of RNA from each sample was used in the RT reaction, conducted according to the instructions of a transcription kit (DRR037A; Takara Biotechnology Co., Ltd.).

Real-time qPCR was used to analyze the mRNA levels of XBP1 or levels of miR-199, with SYBR Premix Ex Taq (Takara Biotechnology Co., Ltd.) on an ABI 7300 system
(Applied Biosystems; Thermo Fisher Scientific, Inc.). The PCR primers used for XBP1, cyclin D and the internal control $\beta$-actin are listed in Table I. The primers for miR-199 were purchased from Guangzhou RiboBio Co., Ltd. (Guangzhou, China). Data analysis was performed via the $2^{-\Delta \Delta \mathrm{Cq}}$ method (20) using the ABI software (V2.4; Thermo Fisher Scientific, Inc.). The results were expressed as the ratio of XBP1 or cyclin D to $\beta$-actin, or miR-199 to U6.

Western blot analysis. The total protein of the liver tissues samples or Hep3B2.1-7 cells was extracted using a Cell lysis buffer for Western and IP (Beyotime Institute of Biotechnology) supplemented with $1 \% \mathrm{mmol} / \mathrm{l}$ phenylmethylsulfonyl fluoride to block protein degradation by endogenous proteases. Supernatant including protein was isolated by centrifugation at $1,200 \mathrm{x} \mathrm{g}$, and protein concentration was determined with a BCA Protein Assay kit (Beyotime Institute of Biotechnology). A total of $40 \mu \mathrm{g}$ protein (per lane) was subjected to $10 \%$ SDS-PAGE, transferred to polyvinylidene fluoride membranes, and incubated with rabbit anti-XBP1 (sc-8015) or anti-CyclinD (sc-8396) $(1: 2,000$ dilution; Santa Cruz Biotechnology, Inc.) at $37^{\circ} \mathrm{C}$ for $16 \mathrm{~h}$ followed by horseradish peroxidase-conjugated secondary antibodies (1:2,000; A0208; Beyotime Institute of Biotechnology). Protein signals were determined with an enhanced chemiluminescence kit (Amersham; GE Healthcare, Chicago, IL, USA) on a Molecular Imager ChemiDoc XRS System (Bio-Rad, Laboratories, Inc., Hercules, CA, USA). Densitometric analysis was conducted with Image J 1.43 (National Institutes of Health, Bethesda, MA, USA). To confirm equal loading, blots were also incubated with primary mouse anti- $\beta$-actin (sc-47778; $1: 2,000$; Santa Cruz Biotechnology, Inc.).

Statistical analysis. SPSS 11.0 software (SPSS, Inc., Chicago, IL, USA) was used for statistical analysis. Data were expressed as the mean \pm standard error of the mean. Differences in measured values among the groups were analyzed by one-way analysis of variance followed by Bonferroni's multiple comparison tests. Differences were considered significant when $\mathrm{P}<0.05$.

\section{Results}

miR-199, XBPl and cyclin D are involved in HCC. Previous results suggest that abnormal expression of numerous biological 

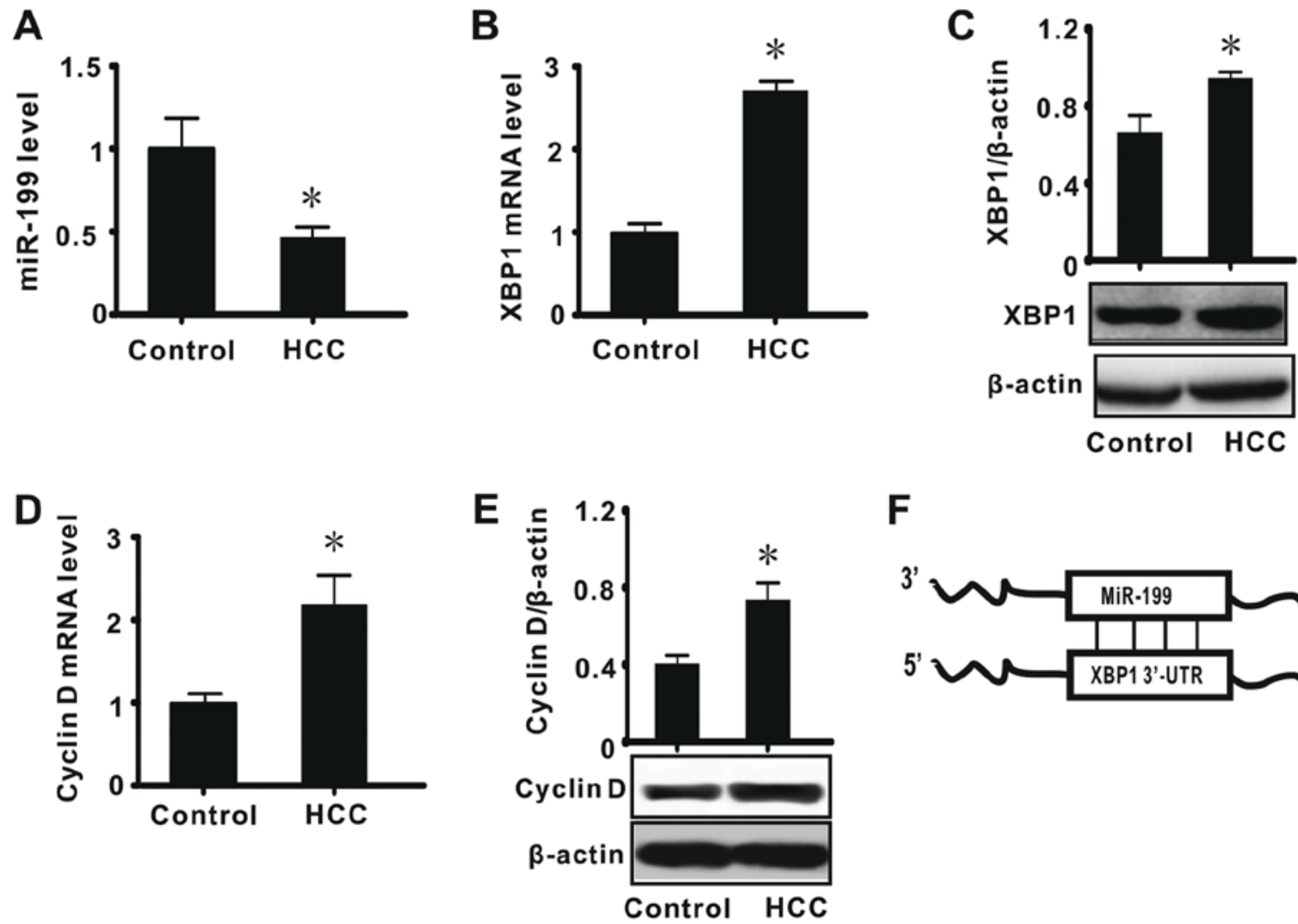

$\mathbf{F}$

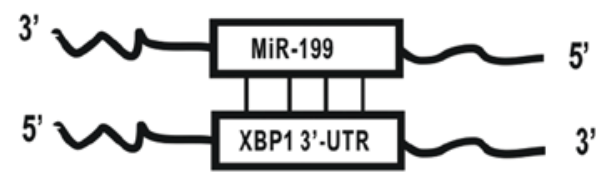

Figure 1. miR-199, XBP1 and cyclin D are involved in HCC. The data show the liver tissue expression levels of (A) miR-199, (B) XPB1 mRNA, (C) XBP1 protein, (D) cyclin D mRNA and (E) cyclin D protein, and (F) the results of bioinformatic analysis. All values were expressed as means \pm standard error of the mean $(n=15)$. miR and mRNA levels were expressed relative to U6 and $\beta$-actin expression, respectively. "P<0.05 vs. control. miR, microRNA; XBP1, X-box binding protein 1; control, normal lung tissue; HCC, hepatocellular carcinoma; UTR, untranslated region.

molecules, including $\alpha$-fetoprotein, is associated with the development of HCC $(1,3,17)$. To investigate whether abnormal expression of miR-199, XBP1 and cyclin D is associated with the pathogenesis of HCC, the expression of these factors was first measured in HCC tumor tissues by real-time RT-qPCR and western blot analysis. The results indicated that miR-199 expression was significantly decreased in tissues diagnosed as HCC when compared with adjacent normal tissues $(\mathrm{P}<0.05$; Fig. 1A). It was also determined that the expression of XBP1 and cyclin $\mathrm{D}$ (mRNA and protein) was significantly increased in HCC $(\mathrm{P}<0.05$; Fig. 1B-E). These results suggested that miR-199, XBP1 and cyclin D may serve important biological functions in HCC. Bioinformatic analysis was subsequently performed to identify the relationship between miR-199 and $\mathrm{XBP} 1$, and the results indicated XBP1to be a direct target of miR-199 (Fig. 1F). Considering miRNA is a negative regulator of gene expression and cyclin D is downstream of XBP1, we speculated that a miR-199/XBP1/cyclin D pathway may have a key role in the development of HCC.

miR-199 directly suppresses the expression of XBP1 by binding with the 3 'untranslated region (UTR) of XBP1. Subsequent experiments were conducted to examine the association between miR-199 and XBP1. Hep3B2.1-7 cells were transfected with miR-199 mimic or inhibitor to increase or decrease the expression of miR-199, respectively. The expression of miR-199 and XBP1 was then measured. It was observed that transfection with miR-199 mimic or inhibitor significantly increased $(\mathrm{P}<0.001)$ or decreased $(\mathrm{P}<0.05)$ the expression level of miR-199; whereas the NC miRNA did not alter the level of miR-199 (Fig. 2A). This confirmed the effects of the miR-199 mimic and inhibitor. The expression of XBP1 (mRNA and protein) was significantly decreased in Hep3B2.1-7 cells treated with miR-199 mimic, and was increased in cells transfected with miR-199 inhibitor, when compared with control cells $(\mathrm{P}<0.05)$ or cells treated with NC miRNA $(\mathrm{P}>0.05)$ (Fig. 2B and C). This preliminarily suggested that miR-199 may negatively regulate the expression of XBP1.

Subsequently, to confirm that miR-199 directly inhibits the expression of XBP1, a luciferase reporter plasmid was constructed containing the seed sequence of the 3'UTR of XBP1 (XBP1-3'UTR-WT; Fig. 2D) or a mutated seed sequence of the XBP1 3'UTR (XBP1-3'UTR-MT). The luciferase reporter plasmids and miR-199 mimic were co-transfected into Hep3B2.1-7 cells and the relative luciferase activity was measured. The miR-199 mimic significantly decreased the relative luciferase activity in cells transfected with XBP1-3'UTR-WT $(\mathrm{P}<0.05$ vs. NC miRNA + XBP1-3'UTR-WT group), but had no effect on the cells treated with XBP1-3'UTR-MT (P>0.05 vs. NC miRNA + XBP1-3'UTR-MT group; Fig. 2E). This suggested that miR-199 directly inhibited the expression of XBP1.

Effect of the inhibition or knockdown of XBPI on the expression of cyclin $D$. In the present study, it was determined that there is an association among miR-199, XBP1 and cyclin D. Considering miR-199 appeared to suppress XBP1, we 
A

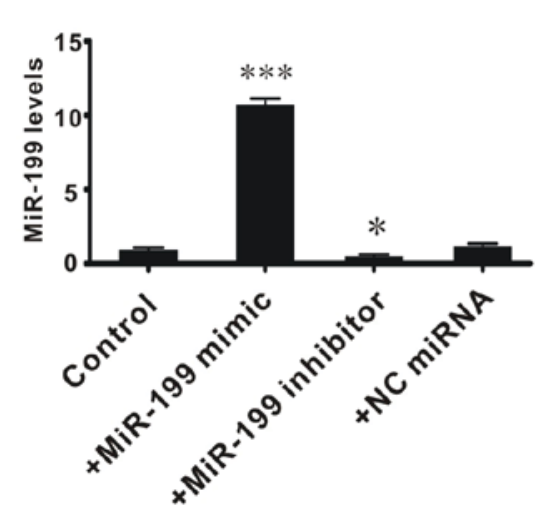

D

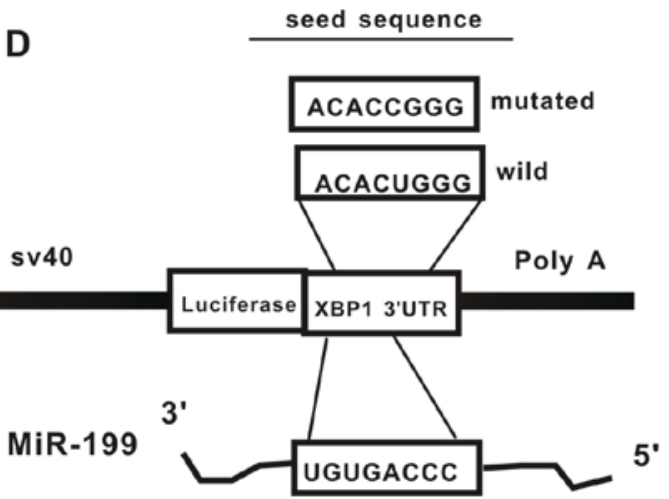

B

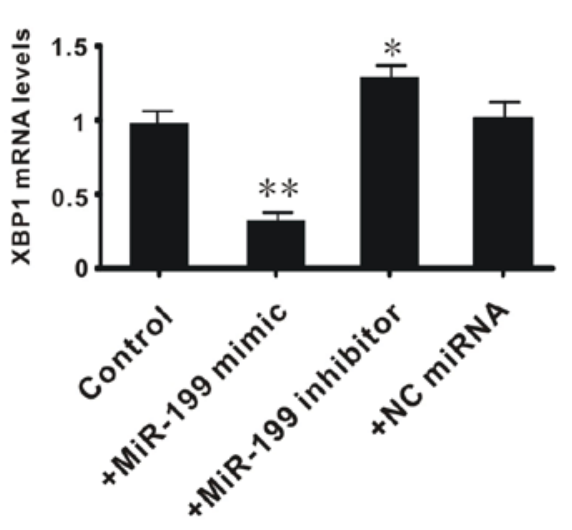

C

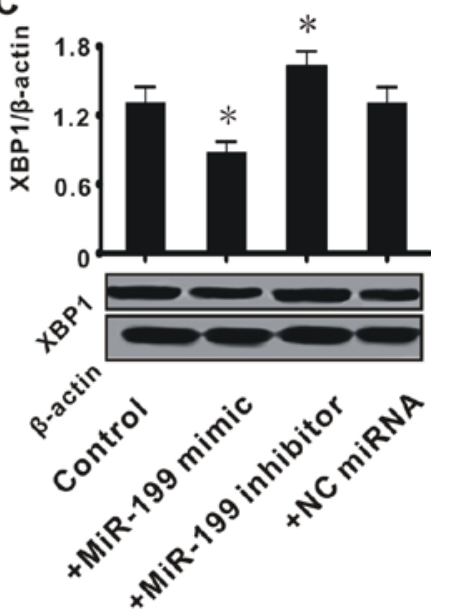

E

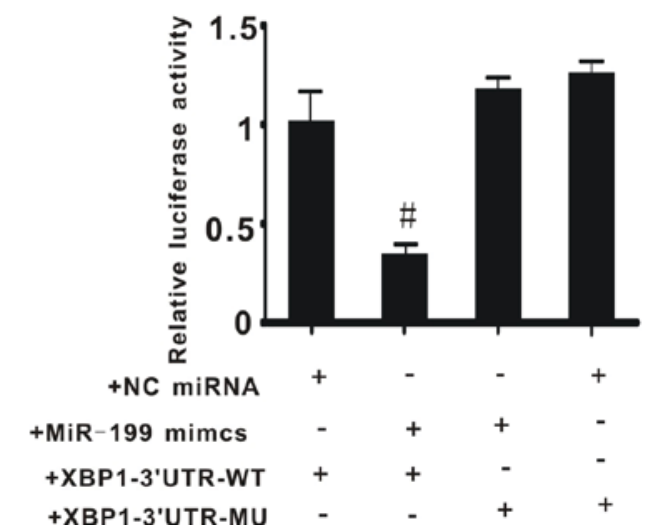

Figure 2. miR-199 directly suppresses the expression of XBP1 by binding with the 3'UTR. (A) miR-199 level; (B) XPB1 mRNA level; (C) XBP1 protein level; (D) schematic diagram of luciferase reporter plasmid construction; (E) relative luciferase activity. All values were expressed as means \pm standard error of the mean. " $\mathrm{P}<0.05$ vs. control; ${ }^{* *} \mathrm{P}<0.01$ and ${ }^{* * * *} \mathrm{P}<0.001$ vs. control; ${ }^{*} \mathrm{P}<0.05$ vs. $\mathrm{NC}$ miRNA + XBP1-3'UTR-WT group. miR, microRNA; XBP1, X-box binding protein 1; NC, negative control; UTR, untranslated region; WT, wild type; MU, mutant.

speculated that the miR-199/XBP1 axis has roles in regulating cyclin D expression. Thus, further experiments were conducted to observe the effect of the inhibition or knockdown of XBP1 on the expression of cyclin D. It was determined that exogenous miR-199 significantly inhibited the expression of cyclin D (mRNA and protein; $\mathrm{P}<0.05$ ), while miR-199 inhibitor upregulated its expression $(\mathrm{P}<0.05$; Fig. $3 \mathrm{~A}$ and $\mathrm{B})$. siRNA against XBP1 significantly suppressed the expression of XBP1 (mRNA and protein; $\mathrm{P}<0.05$ ), while the NC siRNA had no effect (Fig. 3C and D). This confirmed the effect of the siRNA transfection. The effect of XBP1 knockdown on the expression of cyclin D was next observed, and it was found that siRNA against XBP1 significantly decreased the expression of cyclin D (mRNA and protein; P<0.05; Fig. 3E-F). These data suggested that miR-199/XBP1 serve an important role in cell proliferation.

Effect of exogenous miR-199 mimic or inhibitor on the proliferation of Hep3B2.1-7 cells. Previous studies have demonstrated that XBP1 may serve an important role in cell proliferation, and we found that miR-199 directly suppressed the expression of XBP1. Thus, further experiments aimed to investigate the effect of miR-199 on the proliferation of Hep3B2.1-7 cells. Results of EdU and DAPI double-staining indicated that transfection with miR-199 mimic caused a reduction in the number of newly proliferated cells, while treatment with miR-199 inhibitor caused an increase in newly proliferated cells, when compared with controls (Fig. 4A). This indicated that miR-199 inhibited the proliferation of Hep3B2.1-7 cells. Consistent with the results of EdU and DAPI-double staining, the data from the CCK-8 assay showed that overexpression or inhibition of miR-199 significantly decreased or increased cell proliferation at all three time points $(12,24$ and $36 \mathrm{~h} ; \mathrm{P}<0.05)$, respectively; whereas the NC miRNA had no effect (Fig. 4B). These data indicated that miR-199 serves an important role in the proliferation of Hep3B2.1-7 cells.

\section{Discussion}

In the present study, it was demonstrated that miR-199 may serve an important role in HCC. It was observed that low expression of miR-199 in HCC tumor tissues was accompanied by high expression of XBP1 and cyclin D. This suggested that miR-199 may be involved in regulation of the tumor cell cycle. Moreover, the bioinformatics analysis indicated XBP1 to be a putative target of miR-199. A luciferase reporter assay and gain- and lose-function studies of miR-199 demonstrated that miR-199 inhibited the expression of XBP1 by directly 
A

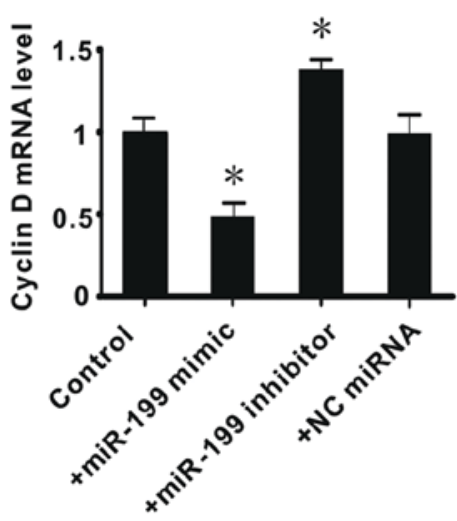

D

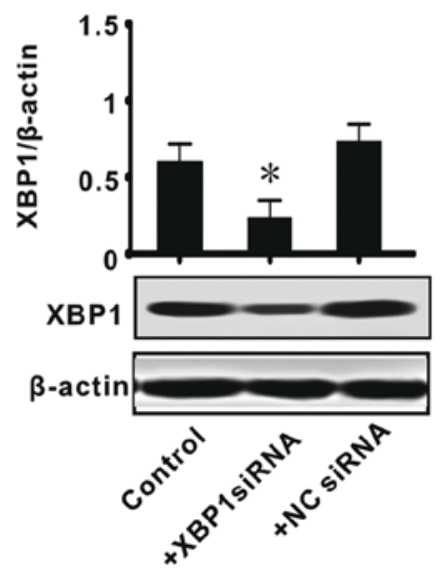

B



$\mathbf{E}$

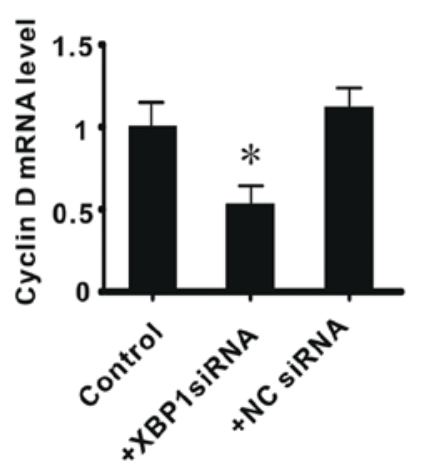

C

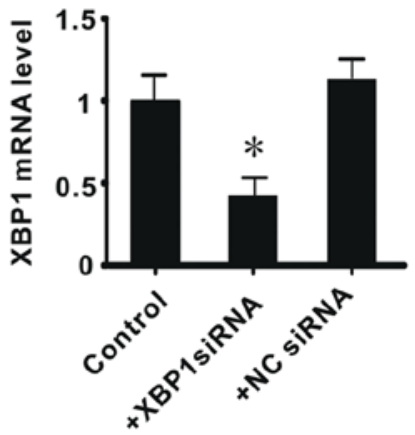

$\mathbf{F}$



Figure 3. Effect of the inhibition or knockdown of XBP1 on the expression of cyclin D. (A) Cyclin D mRNA level; (B) cyclin D protein level; (C) XBP1 mRNA level; (D) XBP1 protein level; (E) cyclin D mRNA level; (F) cyclin D protein level. All values were expressed as means \pm standard error of the mean. ${ }^{*} \mathrm{P}<0.05$ vs. control. MiR, microRNA; XBP1, X-box binding protein 1; siRNA, small interfering RNA; NC, negative control.

binding to its 3'UTR. As stated, the expression level of XBP1 was negatively associated with the expression of miR-199. Knockdown of XBP1 decreased the expression of cyclin D in Hep3B2.1-7 cells. This confirmed cyclin D as a downstream target of XBP1. Furthermore, overexpression of miR-199 markedly inhibited the proliferation of Hep3B2.1-7 cells, whereas inhibition of miR-199 promoted proliferation. Thus the present study, to our knowledge, is the first to demonstrate that a miR-199/XBP1/cyclin D axis may be involved in tumor cell proliferation in of HCC.

HCC is associated with distinct histological and etiological features compared with other forms of primary liver cancer (21), and $70-90 \%$ of patients with HCC have an established background of chronic liver disease and cirrhosis (1). Cirrhosis is a main risk factor for the development of HCC, the major causes of which are hepatitis $\mathrm{B}$ and hepatitis $\mathrm{C}$ viral infection $(22,23)$. Although $30-40 \%$ of patients with HCC are eligible for curative treatments, which include surgical resection as the first-line treatment, liver transplantation and percutaneous ablation, there remains a high frequency of tumor recurrence following surgical resection, and most cases of HCC appear resistant to conventional chemotherapy and radiotherapy $(1,2)$. This suggests that the pathogenesis of $\mathrm{HCC}$ is a complex process. In fact, $\mathrm{HCC}$ carcinogenesis may involve various modifications in a number of molecular pathways as well as genetic alterations, which ultimately lead to malignant transformation and HCC disease progression (24). For example, hepatocellular carcinoma (HCC) has been associated with increased insulin-like growth factor (IGF) signaling, and sorafenib, a multi-tyrosine kinase inhibitor, which directly targets the IGF pathway, is the only chemotherapeutic option for patients with advanced HCC (3). As is known, the IGF system is involved in cellular proliferation in most cell types, particularly in the hyper-proliferation of HCC cells (3), which serves as indication of abnormal proliferation being among the main causative factors of HCC. Therefore, elucidating the mechanism responsible for tumor cell proliferation in HCC may be key for improvement of HCC treatment and novel drug development.

It is established that deregulations of cell cycle genes exist in HCC. For example, retinoblastoma protein is frequently abnormally expressed in many cell types in HCC, and the four main cyclin types (D, E, A and B) have been observed to be overexpressed in HCC and are associated with different outcomes (25). In addition, dysregulation of pleiotropic growth factors, receptors and their downstream signaling pathway components represent a central protumorigenic component of hepatocarcinogenesis (25). In particular, the IGF/IGF-1 receptor, hepatocyte growth factor (HGF)/HGF receptor, Wingless/ $\beta$-catenin, transforming growth factor- $\alpha /$ epidermal growth factor receptor and transforming growth factor (TGF)- $\beta /$ TGF- $\beta$ receptor pathways 
A

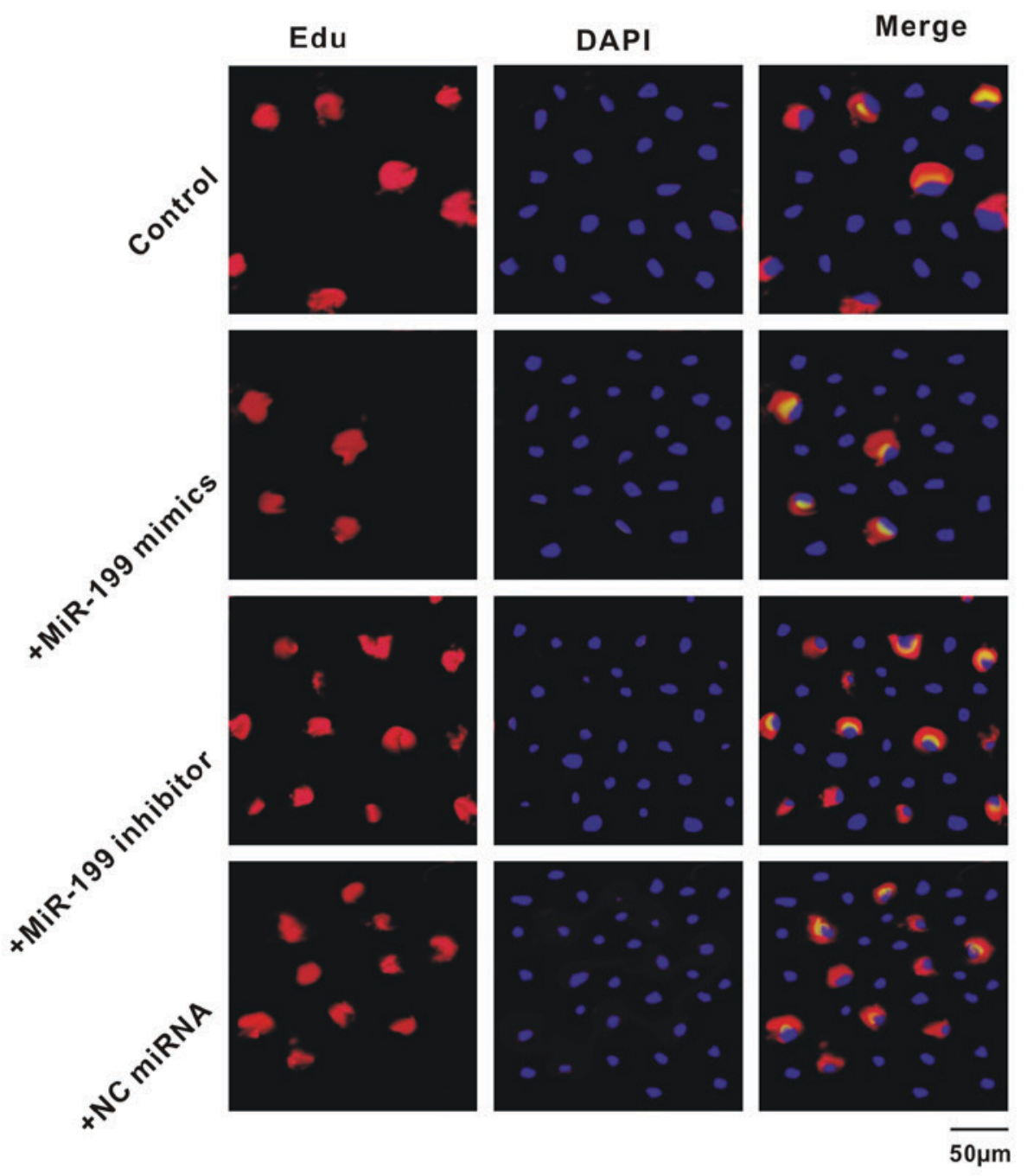

B

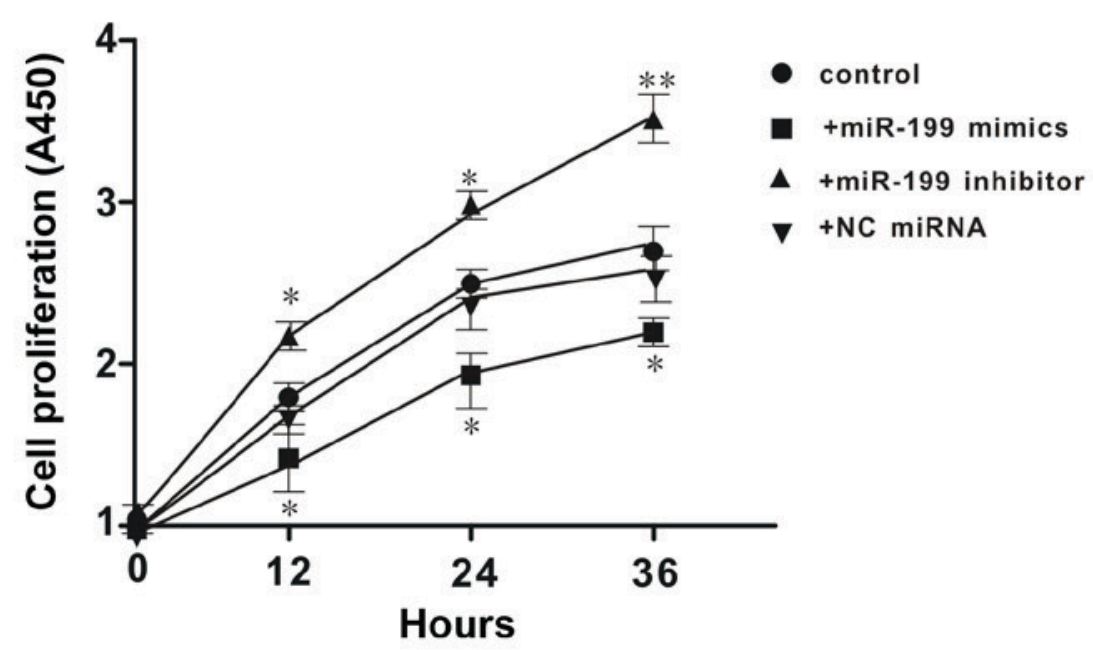

Figure 4. Effect of exogenous miR-199 mimic or inhibitor on the proliferation of Hep3B2.1-7 cells. (A) Representative images of EdU and DAPI double staining (x200); (B) Cell Counting Kit- 8 assay results. All values were expressed as means \pm standard error of the mean. ${ }^{*} \mathrm{P}<0.05$ and ${ }^{* * *} \mathrm{P}<0.01$ vs. control. miR: microRNA; EdU, 5-ethynyl-2'-deoxyuridine; NC, negative control. 
contribute to the proliferative, anti-apoptotic and invasive behaviors of HCC cells (26). However, there is no single mechanism that can fully explain the pathogenesis of tumor cell hyper-proliferation in HCC. Recent studies revealed that XBP1 was closely linked with tumor occurrence $(4,8,10-12)$. Numerous researches have reported that the IRE1/XBP1 pathway is involved in cell survival, epithelial-mesenchymal transition, transcription factor regulation, drug resistance and glycolysis in tumor cells (8-13). In addition, XBP1 has been demonstrated to be necessary for cellular proliferation (27) and its underlying mechanism involves regulation of the expression of matrix metalloproteinase-9 (28) and phosphatidylinositol-4,5-bisphosphate 3-kinase/mechanistic target of rapamycin (29). As is known, XBP1 is widely expressed in various tissues in mammals, particularly to a high level in the liver, where it serves as a necessary factor for liver cell differentiation and tissue formation (8). Therefore, it may be deduced that XBP1 is an important molecule responsible for HCC cell proliferation. Indeed this was indicated by the present experiments, since miR-199 inhibition and therefore increased expression of XBP1 was positively associated with the proliferation of Hep3B2.1-7 cells.

Recent data have shown that epigenetic alteration is an important cause of the development and progression of HCC, with alterations including DNA methylation, acetylation and post-transcriptional regulation of miRNAs (30). Among these, dysregulation of miRNAs has been considered as one of the most attractive factors (as they can be used as disease diagnosis biomarkers) associated with the pathogenesis of HCC, with reports suggesting that miRNAs serve critical roles in HCC progression. For example, deregulation of miRNA may lead to abnormal cell proliferation, evasion of apoptotic cell death, angiogenesis, and invasion and metastasis, by targeting a number of protein-coding genes (30). Moreover, several studies on the role of miRNAs in cell proliferation have found that mir-503a, mir-138, mir-199 and mir-214 were involved in regulation of the cell cycle in HCC cells (16-19). Consistently, the present study and other studies $(19,31)$ have determined that miR-199 expression was significantly decreased in HCC tumor cells. Furthermore, in the current study, it was indicated that low expression of miR-199 contributed to the hyper-proliferation of Hep3B2.1-7 cells by directly suppressing XBP1. Taken together, these data indicate that the miR-199/XBP1 pathway serves an important role in HCC.

Numerous studied are required to observe the role of miR-199/XBP1 in HCC, particularly a study investigating of its role in vivo, including observing the effect of miR-199 on tumorigenesis when the Hep3B2.1-7 cells were injected into nude mice. However, to our knowledge, the present study is the first to demonstrate that low expression of miR-199 was associated with HCC, and that the underlying mechanism of miR-199 involved direct suppression of XBP1. This may provide novel theoretical foundation for understanding the molecular mechanisms of HCC, as well as novel targets for HCC treatment and drug development.

\section{Acknowledgements}

Not applicable.

\section{Funding}

The present work was supported by the HuNan Provincial Science \& Technology Department of the Affiliated Changsha Hospital of Hunan Normal University (grant no. 2018JJ6126 to $\mathrm{GHH}$ ) and the Education Department of HuNan Province of Changsha Medical University (grant no. 15C0161 to ZL).

\section{Availability of data and materials}

The datasets used and/or analyzed during the present study are available from the corresponding author on reasonable requires.

\section{Authors' contributions}

ZL, YQG and XZ contributed to the acquisition of data. GHH contributed to the design of the experiment and drafted the manuscript. All authors have read and approved the manuscript.

\section{Ethics approval and consent to participate}

The use of materials in the present study was approved by the Hunan Normal University Medical Ethics Committee, and all patients had signed written informed consent.

\section{Patient consent for publication}

Patients were informed and consented to the publication of this manuscript.

\section{Competing interests}

The authors declare that they have no competing interests.

\section{References}

1. Sanyal AJ, Yoon SK and Lencioni R: The etiology of hepatocellular carcinoma and consequences for treatment. Oncologist 15(Suppl 4): S14-S22, 2010.

2. Pievsky D and Pyrsopoulos N: Profile of tivantinib and its potential in the treatment of hepatocellular carcinoma: The evidence to date. J Hepatocell Carcinoma 3: 69-76, 2016.

3. Enguita-Germán M and Fortes P: Targeting the insulin-like growth factor pathway in hepatocellular carcinoma. World $\mathrm{J}$ Hepatol 6: 716-737, 2014.

4. Feitelson MA, Arzumanyan A, Kulathinal RJ, Blain SW, Holcombe RF, Mahajna J, Marino M, Martinez-Chantar ML, Nawroth R, Sanchez-Garcia I, Sharma D, et al: Sustained proliferation in cancer: Mechanisms and novel therapeutic targets. Semin Cancer Biol 35: S25-S54, 2015.

5. Derenzini M, Trerè D, Pession A, Govoni M, Sirri V and Chieco P: Nucleolar size indicates the rapidity of cell proliferation in cancer tissues. J Pathol 191: 181-186, 2000.

6. López-Sáez JF, de la Torre C, Pincheira J and Giménez-Martín G: Cell proliferation and cancer. Histol Histopathol 13: 1197-1214, 1998.

7. Glimcher LH: XBP1: The last two decades. Ann Rheum Dis 69(Suppl 1): i67-i71, 2010.

8. Marumoto Y, Terai S, Urata Y, Matsumoto T, Mizunaga Y, Yamamoto N, Jin H, Fujisawa K, Murata T, Shinoda K, et al: Continuous high expression of XBP1 and GRP78 is important for the survival of bone marrow cells in CCl4-treated cirrhotic liver. Biochem Biophys Res Commun 367: 546-552, 2008.

9. Chen X, Iliopoulos D, Zhang Q, Tang Q, Greenblatt MB, Hatziapostolou M, Lim E, Tam WL, Ni M, Chen Y, et al: XBP1 promotes triple-negative breast cancer by controlling the HIF1 $\alpha$ pathway. Nature 508: 103-107, 2014. 
10. Ming J, Ruan S, Wang M, Ye D, Fan N, Meng Q, Tian B and Huang T: A novel chemical, STF-083010, reverses tamoxifen-related drug resistance in breast cancer by inhibiting IRE1/XBP1. Oncotarget 6: 40692-40703, 2015.

11. Liu Y, Hou X, Liu M, Yang Z, Bi Y, Zou H, Wu J, Che H, Li C, Wang X, et al: XBP1 silencing decreases glioma cell viability and glycolysis possibly by inhibiting HK2 expression. J Neurooncol 126: 455-462, 2016.

12. Chae U, Park SJ, Kim B, Wei S, Min JS, Lee JH, Park SH, Lee AH, Lu KP, Lee DS and Min SH: Critical role of XBP1 in cancer signalling is regulated by PIN1. Biochem J 473: 2603-2610, 2016.

13. Sun H, Lin DC, Guo X, Kharabi Masouleh B, Gery S, Cao Q, Alkan S, Ikezoe T, Akiba C, Paquette R, et al: Inhibition of IRE1 $\alpha$-driven pro-survival pathways is a promising therapeutic application in acute myeloid leukemia. Oncotarget 7 : 18736-18749, 2016.

14. Diehl JA, Fuchs SY and Koumenis C: The cell biology of the unfolded protein response. Gastroenterology 141: 38-41, 41.e1-2, 2011.

15. Zhang D and Richardson DR: Endoplasmic reticulum protein 29 (ERp29): An emerging role in cancer. Int J Biochem Cell Biol 43: 33-36, 2011.

16. Greene CM, Varley RB and Lawless MW: MicroRNAs and liver cancer associated with iron overload: Therapeutic targets unravelled. World J Gastroenterol 19: 5212-5226, 2013.

17. Xiao F, Zhang W, Chen L, Chen F, Xie H, Xing C, Yu X, Ding S, Chen K, Guo H, et al: MicroRNA-503 inhibits the G1/S transition by downregulating cyclin D3 and E2F3 in hepatocellular carcinoma. J Transl Med 11: 195, 2013.

18. Wang W, Zhao LJ, Tan YX, Ren H and Qi ZT: MiR-138 induces cell cycle arrest by targeting cyclin D3 in hepatocellular carcinoma. Carcinogenesis 33: 1113-1120, 2012.

19. Duan Q, Wang X, Gong W, Ni L, Chen C, He X, Chen F, Yang L, Wang P and Wang DW: ER stress negatively modulates the expression of the miR-199a/214 cluster to regulates tumor survival and progression in human hepatocellular cancer. PLoS One 7: e31518, 2012

20. Livak KJ and Schmittgen TD: Analysis of relative gene expression data using real-time quantitative PCR and the 2(-Delta Delta C(T)) method. Methods 25: 402-408, 2001.

21. Yu MC, Yuan JM and Lu SC: Alcohol, cofactors and the genetics of hepatocellular carcinoma. J Gastroenterol Hepatol 23 (suppl 1): S92-S97, 2008
22. El-Serag HB and Rudolph KL: Hepatocellular carcinoma: Epidemiology and molecular carcinogenesis. Gastroenterology 132: 2557-2576, 2007.

23. Poon D, Anderson BO, Chen LT, Tanaka K, Lau WY, Van Cutsem E, Singh H, Chow WC, Ooi LL, Chow P, et al: Management of hepatocellular carcinoma in Asia: Consensus statement from the Asian Oncology Summit 2009. Lancet Oncol 10: 1111-1118, 2009.

24. Monga SP: Role of Wnt// -catenin signaling in liver metabolism and cancer. Int J Biochem Cell Biol 43: 1021-1029, 2011.

25. Bisteau X, Caldez MJ and Kaldis P: The complex relationship between liver cancer and the cell cycle: A story of multiple regulations. Cancers (Basel) 6: 79-111, 2014

26. Breuhahn K, Longerich $\mathrm{T}$ and Schirmacher P: Dysregulation of growth factor signaling in human hepatocellular carcinoma. Oncogene 25: 3787-3800, 2006.

27. Hasegawa D, Calvo V, Avivar-Valderas A, Lade A, Chou HI, Lee YA, Farias EF, Aguirre-Ghiso JA and Friedman SL: Epithelial $\mathrm{Xbp} 1$ is required for cellular proliferation and differentiation during mammary glanddevelopment. Mol Cell Biol 35: $1543-1556,2015$.

28. Xia T, Tong S, Fan K, Zhai W, Fang B, Wang SH and Wang JJ: XBP1 induces MMP-9 expression to promote proliferation and invasion in human esophagealsquamous cell carcinoma. Am J Cancer Res 6: 2031-2040,2016.

29. Yang J, Cheng D,Zhou S,Zhu B,Hu T and Yang Q: Overexpression of X-Box binding protein 1 (XBP1) correlates to poor prognosis and up-regulation of PI3K/mTOR in human osteosarcoma. Int J Mol Sci 16: 28635-28646, 2015.

30. Li CW, Chang PY and Chen BS: Investigating the mechanism of hepatocellular carcinoma progression by constructing geneticand epigenetic networks using NGS data identification and big database mining method. Oncotarget 7: 79453-79473, 2016.

31. El-Abd NE, Fawzy NA, El-Sheikh SM and Soliman ME: Circulating miRNA-122, miRNA-199a, and miRNA-16 as biomarkers for early detection of hepatocellular carcinoma in egyptian patients with chronic hepatitis c virus infection. Mol Diagn Ther 19: 213-220, 2015.

This work is licensed under a Creative Commons Attribution-NonCommercial-NoDerivatives 4.0 International (CC BY-NC-ND 4.0) License. 